Rev. salud pública. 10 sup (1): 72-82, 2008

Ensayos/Essays

\title{
El Concepto de Equidad y el Debate sobre lo Justo en Salud
}

\author{
The concept of equity and the debate about fairness in health
}

\author{
Mario Hernández-Álvarez \\ Universidad Nacional de Colombia, Bogotá. mehernandeza@unal.edu.co \\ Recibido 2 Marzo 2008/Enviado para Modificación 31 Mayo 2008/Aceptado 2 Octubre 2008
}

\section{RESUMEN}

El ensayo presenta la propuesta de entender la equidad en salud en el marco de la discusión ético-política de la justicia, para lo cual se revisa el debate actual y se muestran las implicaciones en la definición de políticas públicas y en la organización de los sistemas de servicios de salud. En segunda instancia se presenta la idea de la historicidad del debate y de sus realizaciones en las sociedades contemporáneas, de manera que el predominio de una u otra posición es un resultado histórico que depende de las relaciones de poder y la correlación de fuerzas en el interior de cada sociedad, en el marco de la interdependencia global. Con base en lo anterior, se sugiere un proceso de construcción democrática para avanzar en una nueva configuración de la equidad en salud para los países latinoamericanos.

Palabras Clave: Equidad en salud, sistemas de salud, justicia sanitaria (fuente: DeCS, BIREME).

\section{ABSTRACT}

This work deals with understanding equity in health within the framework of an ethicalpolitical discussion of justice; current debate on this issue is reviewed and the implications in defining public policy and organising health service systems are shown. The debate's historicity and how it is carried out in contemporaneous societies is presented so that one or another position's predominance is a historical result depending on power relationships and correlating efforts within each society, within the framework of global interdependency. A process of democratic construction is thus suggested, based on the foregoing, for advancing towards a new configuration of equity in health for Latin-American countries.

Key Words: Equity in health, health system, sanitary justice (source: MeSH. NLM).

$\mathrm{P}$ ara hablar de equidad en salud es necesario hacer explícito qué se entiende por "salud” y qué por “equidad”. El argumento central de este ensayo parte de aceptar que social e históricamente existen y han existido muchas maneras de entender la salud y la equidad, y no son asuntos 
resueltos. Las distintas posiciones conviven en conflicto en el interior de las sociedades, en medio de la interdependencia global.

Equidad no es lo mismo que igualdad. Equidad implica valorar las desigualdades desde una idea de justicia. El tipo de valoración utilizado tiene implicaciones políticas, tanto en las decisiones cotidianas como en las políticas públicas. Existen relaciones profundas entre las posiciones éticas y los desarrollos científicotécnicos que sustentan las políticas, de manera que no puede hablarse de neutralidad técnica en esta materia, a pesar del esfuerzo de los técnicos. Además, el tipo de valoración predominante es un resultado histórico de cada sociedad. En medio de la correlación de fuerzas, se producen predominios de unas posiciones sobre otras, a veces de manera hegemónica e imperceptible. Así, la equidad en salud expresa la justicia social predominante en una sociedad. Frente a situaciones consideradas problemáticas en materia de equidad en salud, como las actuales, vale la pena hacer explícitas las posiciones en debate y arriesgar, con mucho realismo, la construcción de nuevas alternativas.

Las definiciones y los límites

Las sociedades han construido diferentes formas de entender la salud y la enfermedad. Si bien, desde el siglo XIX, existe un gran predominio del discurso científico-técnico para definirlas, tal discurso no es aceptado por todos los grupos y comunidades, y tampoco es un discurso unívoco. En su interior existen controversias bastante reconocidas. Dependiendo del tipo de concepción sobre lo sano y lo enfermo, el debate sobre equidad en salud queda más o menos delimitado.

No es objeto de este ensayo profundizar en el tema de los diferentes conceptos de salud, pero sí llamar la atención sobre los límites: es muy distinto hablar de equidad cuando se piensa en salud como la ausencia de enfermedad y por lo tanto, el eje de análisis se centra en los servicios de atención en salud, que cuando se piensa en el desarrollo de proyectos de vida, y el eje tiende a ser las trayectorias y las condiciones de vida. Tampoco es lo mismo concebir la salud y la enfermedad como fenómenos naturales distribuidos entre las poblaciones de manera probabilística o aleatoria en función de grupos de factores, o pensar que la enfermedad es un fenómeno social y, por lo tanto, el orden biológico queda subsumido jerárquicamente en el orden social (1). El debate actual sobre causalidad probabilística o jerárquica de la salud y la enfermedad de las poblaciones se relaciona con la manera de entender las inequidades en salud, su generación y su superación (2). 
Del lado del concepto de equidad, el asunto no parece resolverse con una definición. Por ejemplo, Barry planteaba en la década del 60 que la equidad es un concepto comparativo entre grupos humanos y se realizaría cuando se lograra " que los iguales sean tratados de igual forma y que los desiguales sean tratados en forma desigual " (3). Esta propuesta fue adoptada más tarde en salud por Culyer y Wagstaff (4) como las dos dimensiones de la equidad: horizontal y vertical. Pero cabe preguntarse quién y cómo define el parámetro de referencia o si la «igualdad» y la «desigualdad» de las personas son asuntos naturales y tan evidentes que no requieren discusión. Aún en el caso de las desigualdades sexuales o propias del ciclo vital, la equidad podría resolverse guardando la proporcionalidad natural o social, sin producir cambio alguno.

En el caso de la famosa definición de equidad en salud de Margareth Whitehead, como "la ausencia de desigualdades innecesarias, injustas y evitables” (5), cabe preguntarse quién y cómo define lo innecesario, lo injusto y lo evitable. O si se asume la definición propuesta por Braveman y Guskin como "la ausencia de disparidades sistemáticas en salud (o sus determinantes) entre grupos sociales más o menos aventajados, en función de la riqueza, el poder o el prestigio” (6), ¿'significa esto que la equidad implica igualdad de riqueza, poder y prestigio entre los grupos sociales o la ausencia de estratificación social? ¿Cuál es el ideal de sociedad justa que sustenta esta perspectiva?

De lo anterior, se obtienen por lo menos dos conclusiones: primero, que la equidad se relaciona con alguna manera de entender lo justo, y la justicia no parece ser un principio unívoco. Segundo, la manera de entender lo justo tiene implicaciones en la acción individual y colectiva, es decir, tiene consecuencias políticas.

El debate inconciente sobre lo justo en salud: posiciones e implicaciones

Aristóteles propuso entender la justicia en dos niveles: la justicia conmutativa, aplicable a las relaciones entre iguales; y la justicia distributiva, cuando alguien con el poder para hacerlo (el gobernante) debe repartir algún bien entre otros. En éste último caso, para Aristóteles la distribución más justa sería aquella que diera a cada quien "lo suyo", esto es, lo que le corresponde en función de su «lugar natural». Para los clásicos griegos, los seres ocupaban un lugar en la naturaleza que los hacía, en sí mismos, desiguales. La Phycis, como se denominaba a la fuerza creadora natural, tendía a preservar el equilibrio, de manera que las desigualdades hacían parte de la perfección natural. Así, la distribución más justa sería aquella que lograra preservar la "proporcionalidad natural” (7), en función 
de las virtudes desiguales de las personas expresadas en la jerarquía social, y no sería deseable ni conveniente una atención igual para todos y todas, sino proporcional al rango y la estratificación social.

Durante toda la Edad Media, el cristianismo desplazó la idea de Physis por la de la voluntad de un solo Dios verdadero, de manera que la distribución proporcional al lugar dado por Dios a los hombres en la tierra, siguió entendiéndose como la más justa. Si el cristianismo desarrolló el cuidado de los enfermos no fue en función de un principio de justicia, sino de conmiseración con el sufrimiento del otro, considerado como "hermano en Dios". Desde esta perspectiva aristotélica clásica, cristiana o neoaristotélica, como se ha intentado rescatar (8), ni los servicios de salud, ni las condiciones de vida deben ser brindados de igual manera. Por esta vía, no serían injustas las desigualdades derivadas de la estratificación social y no debería haber una intervención contra natura. No es difícil encontrar defensores de esta posición en la actualidad, aunque no se haga conciencia de ello.

La separación entre los órdenes natural, humano y divino, propiciada por el humanismo renacentista y después por el protestantismo anglosajón, condujo a John Locke a su planteamiento central de justicia como "libertad contractual" en el siglo XVII. Retomando viejas discusiones teológicas, Locke consideraba el libre albedrío como un don divino que fatalmente separa al hombre de la voluntad de Dios. El trabajo para subsistir y la libertad para elegir, serían condiciones innatas, producto de la creación. De allí que Locke propusiera la libertad, la propiedad (producto del trabajo y la transacción) y la integridad personal para trabajar como derechos humanos inalienables. Si se otorga poder al gobernante, a través del “contrato social”, sólo debe ser para garantizar tales derechos. Nada más. Cualquier otra intervención no sólo es injusta sino inmoral. En sentido estricto, estos derechos deberían ser garantizados con el monopolio de la fuerza y algún tipo de justicia conmutativa que hiciera valer los contratos, esto es, las transacciones entre "hombres libres e iguales", o bien restituyera las apropiaciones injustas, por robo o agresión personal (7).

El planteamiento liberal no es una vieja historia del siglo XVII. Su rescate le significó a Robert Nozick ganar el premio Pulitzer en Estados Unidos por su best seller titulado Anarchy, State, and Utopia en 1974 (9). El desarrollo filosófico de Nozick sirvió de fundamento moral a las propuestas de Hayeck y Freedman, y se convirtió en una de las vías radicales del neoliberalismo en los años 70. La consecuencia en salud de esta posición consiste en creer que no existe ninguna justificación moral para considerar los servicios de salud como derechos huma- 
nos ni, por lo tanto, deberes del Estado. Los servicios deben ser obtenidos en la libre competencia de mercado y la capacidad de pago de las personas, en el marco de las transacciones justas, expresadas en contratos garantizados por el Estado. Si se quisiera brindar servicios a los pobres, esto sólo sería basado en el sentimiento de conmiseración que genera la caridad voluntaria o la filantropía, y no en el principio de justicia (10).

El centro de la crítica de Marx a la posición liberal estaba en el reconocimiento de la desigualdad entre los seres humanos. De hecho, la situación de los obreros ingleses en las fábricas de Manchester a mediados del siglo XIX no podría considerarse igual a la del dueño de la fábrica. Tampoco podía entenderse como el producto de la libre elección de los obreros. De manera que la idea de los contratos entre hombres libres e iguales no correspondía a la realidad. La clave de esa desigualdad estaba en la apropiación de los medios de producción. Mientras el propietario tenía la tierra, la máquina y el dinero para comprar la fuerza de trabajo de los trabajadores, éstos sólo tenían su cuerpo y sus capacidades. La relación contractual no hacía más que alienar al trabajador y expropiarle su producción. De allí que la relación misma resultara productora de desigualdad. La propuesta del Manifiesto Comunista no es otra que la abolición de la propiedad privada, para hacer valer el trabajo de cada cual como aporte a la riqueza colectiva. El problema de la repartición de esa riqueza tendría que acudir a un principio de justicia distributiva, para lo cual Marx retomó a Louis Blanc, uno de los "socialistas utópicos" franceses, quien propuso la máxima "a cada uno debe exigírsele según su capacidad, y debe dársele según sus necesidades”, como orientación para la justa distribución (7).

En salud, la máxima socialista se realizó a través de la implantación de servicios de salud de puertas abiertas, regionalizado y por niveles, para atender a las personas según su necesidad. El componente del aporte según la capacidad se consideraba implícito, dada la condición de trabajador.

En cierta forma, el reconocimiento de los aportes proporcionales a la seguridad social durante el siglo XX, en el marco de la relación salarial, se apoyó en la máxima socialista, pero desde un fundamento ético que no partía de la abolición de la propiedad privada. La confrontación capital-trabajo que se desarrolló en todo el mundo con particularidades nacionales durante el siglo XX, llegó a la expresión socialdemócrata de los derechos sociales, económicos y culturales (DESC) definidos en el pacto de la segunda postguerra en 1948. 
La argumentación moral que sustentó la idea de la ampliación de los derechos humanos es la de reconocer que para ejercer la libertad, es necesario contar con condiciones y medios. Los derechos negativos o de primera generación (vida o integridad física, libertad, propiedad) garantizarían la "libertad de" ser. Los derechos positivos, de segunda generación (los DESC) permitirían la "libertad para” hacer (7). Con base en esta concepción, los servicios de salud se entienden como un derecho ciudadano y un deber del Estado, limitado por los recursos económicos disponibles. A mediados de siglo, la experiencia acumulada de los seguros sociales y de la organización de servicios financiados por impuestos aparecerían como los modelos a seguir, en el marco de la formación de los Estados de Bienestar.

El incremento exponencial de los costos de la atención en salud, en medio de la llamada “crisis de los Estados de Bienestar", reanimó la discusión sobre lo justo en salud durante la década del 70. La posición utilitarista, planteada en el siglo XIX por Bentham y Mill y desarrollada en Economía durante el siglo XX, ocupó un lugar preponderante como fundamento moral de la asignación de los recursos públicos. Para los utilitaristas, cualquier distribución debería demostrar, en sus consecuencias o resultados y frente a una cantidad limitada de recursos, la máxima satisfacción de deseos para el mayor número de individuos y al menor costo posible. De otra forma, la distribución sería injustificable (11). El criterio de "maximimizar las potencialidades individuales", hasta llegar a la "utilidad marginal”, por encima de la cual no habría mayor impacto (óptimo de Pareto), se convirtió en el foco de la gestión de los recursos públicos.

En salud, el cálculo de costo-beneficio y la identificación de prioridades a partir de una valoración de las pérdidas, denominada "carga de la enfermedad" o "años de vida potencialmente perdidos" o "años de vida ajustados a la discapacidad”, se convirtió en la mejor expresión de esta posición, como se observa en el informe Invertir en salud del Banco Mundial de 1993 (12). Esta expresión del utilitarismo en salud, denominada por Peter y Evans como la "visión de prioridad” (2), fue asumida por los organismos internacionales de salud desde los años $90(13,14,15)$ y se utilizó para la evaluación del desempeño de los sistemas de salud en el Informe de Salud en el Mundo del año 2000 (16).

En debate con el utilitarismo, Rawls presentó su Teoría de la justicia en 1971 (17). En esa obra, Rawls asumió una posición liberal, pero desde un “constructivismo kantiano” (18) según el cual, los seres humanos somos sujetos morales racionales, capaces de concebir la sociedad como un sistema imparcial (fair) de cooperación. De allí que proponga una justicia pocedimental, más que 
de igualdad en los resultados. La imparcialidad resultaría entonces de la aplicación de reglas que permitan, primero, contar con ciertas "libertades básicas" o "bienes primarios sociales” para todos y todas, como punto de partida. Y segundo, garantizar que cualquier forma de distribución desigual genere beneficios para los menos aventajados de la sociedad (19).

En salud se ha planteado que esta posición se expresa en la decisión de focalizar sólo en los que están peor, pero no es así. La focalización en los más pobres o en poblaciones priorizadas resulta de una posición utilitarista. Por el contrario, se trataría de avanzar en la definición explícita de servicios de atención y prevención necesarios para la vida humana, como propone Daniels (20), o de un mínimo decente de "bienes esenciales" en salud, como propone Fried (21). En el debate reciente sobre equidad en salud, la posición rawlsiana implica establecer las relaciones entre las inequidades en salud y las injusticias en la estructura básica de la sociedad, entendida como un sistema imparcial de cooperación (2). De allí que la perspectiva de los "determinantes sociales de la salud" se concentre en la identificación y afectación de las “causas de las causas” y en las rutas o procesos de producción de las inequidades, como centro de su análisis y de sus recomendaciones (22).

Cuando Sen discute con Rawls sobre el concepto de libertades básicas, lo hace para llamar la atención sobre el peligro de convertir tales "libertades" en un conjunto de bienes y servicios tangibles cuyo cálculo terminaría en la perspectiva utilitarista del consumo individual. Por esto, Sen prefiere hablar de "capacidades" humanas y de una justicia distributiva capaz de brindar igualdad de "oportunidades» para desarrollar esas capacidades (23). Incluso, Sen advierte sobre la necesidad de contar con la "perspectiva interna” o la "percepción” de los grupos humanos involucrados en las demandas por salud y por equidad en salud, lo que hace más complejo el problema de la distribución, pero también, más respetuoso y democrático (24).

En medio del debate, Alain Enthoven promovió su modelo de “competencia administrada" como alternativa de articulación entre eficiencia y equidad en los sistemas de servicios de salud. Basado en Arrow, con quien trabajó en la década del 50, Enthoven reconocía las asimetrías de los mercados de servicios de salud y aceptaba la necesidad de la intervención de un tercer agente. Pero no encontraba equitativo el mercado de seguros de salud ni la intervención directa del Estado a través del pago por servicios en los tradicionales Medicare y Madicaid en Estados Unidos. Propuso incorporar mecanismos de regulación del mercado de aseguramiento, de manera que los competidores no estuvieran estimulados 
por el precio de la póliza, sino por el número de afiliados y una mayor eficiencia en la operación, preservando la libre elección del usuario y del prestador (25). De allí que Restrepo considere que su propuesta resuelve la tensión entre equidad y eficiencia, pues logra el acceso igual a un conjunto mínimo de servicios (plan de beneficios), con un "costo razonable", que se alcanzaría cuando "los beneficios marginales sean iguales al costo marginal para personas con un ingreso medio" (26). Con esto. Enthoven adoptaba una combinación compleja entre la posición rawlsiana de los bienes primarios básicos y el utilitarismo del cálculo del menor costo y el mayor beneficio.

La revisión de posiciones morales y sus implicaciones en el ordenamiento de las políticas y sistemas de salud puede ser interminable. Pero se trata aquí de mostrar que no es una discusión vana. No se trata de un ejercicio de erudición, sino de maneras de entender y asumir lo justo en materia de salud, en permanente confrontación y con predominios de unas sobre otras en cada sociedad. La conciencia del debate permite, por lo menos, obtener dos conclusiones: primero, considerar que existen alternativas en juego en el debate contemporáneo y, por lo tanto, no es un asunto ya resuelto por los economistas de la salud; y segundo, que las sociedades construyen históricamente sus opciones, de manera que tampoco se trata de tendencias inevitables, aunque existan hegemonías y predominios.

Las tendencias históricas y las particularidades

Cada una de las posiciones morales presentadas ha sido el producto de un proceso histórico. No habría un Locke sin el parlamento inglés del siglo XVII, ni un Marx sin las revoluciones obreras europeas de 1848. Pero más allá de las posiciones, han ocurrido complejos procesos sociales que han generado los cambios, planeados y no planeados, que Elias denomina “figuraciones humanas” (27). El Estado Benefactor europeo no resultó solamente de la adopción de una posición socialdemócrata sobre la justicia social. Fue un complejo proceso que ha tenido varios intentos de comprensión y explicación. Para De Swaan, por ejemplo, el Estado de Bienestar es una particular "configuración a cuatro bandas” en cada país, producto de la correlación de fuerzas entre los grandes empresarios, los trabajadores organizados, la pequeña burguesía y los regímenes políticos de los estados nacionales del período entre guerras, tanto en Europa como en Norteamérica (28).

Polanyi, por su parte, prefiere entender el surgimiento del Estado de Bienestar como una defensa de las sociedades a los efectos deletéreos del mercado autorregulador construido durante el largo siglo XIX y que vio su mayor crisis en 
la Primera Guerra Mundial (29). Castel prefiere hablar de un proceso que va del "contrato" al "estatuto", gracias a la salarización progresiva de las sociedades europeas durante el siglo XX, en medio del conflicto capital-trabajo (30). Y Navarro sustenta su explicación en el grado de poder de la clase obrera, como el elemento determinante del alcance y los tipos de Estados de Bienestar desarrollados en el mundo capitalista (31).

Este proceso no se produjo en los países latinoamericanos. Difícilmente puede hablarse de Estado de Bienestar en América Latina. En materia de servicios de salud, sólo Costa Rica desarrolló un sistema de seguridad social de amplia cobertura y Chile avanzó en la conformación de un servicio único de salud al estilo inglés desde la década del 50. La mayoría de países sostuvo una fragmentación de diferentes proporciones entre el seguro social para trabajadores formales, la asistencia pública para pobres y un sector privado con diferentes formas de pago de bolsillo para los pudientes (32). Este resultado diferente resulta del proceso sociopolítico de configuración del Estado nacional en cada país (33). Es imposible en poco espacio mostrar tales especificidades. Pero el punto crucial está en que las sociedades latinoamericanas, en su mayoría, no han tenido la experiencia vital de la garantía universal de derechos sociales, económicos y culturales, en función de la condición de ciudadano, y esto se traduce en el predominio de la perspectiva contractualista liberal que conduce al sálvese quien pueda.

En estas circunstancias, no es extraño que el modelo del "pluralismo estructurado” (34), basado en la competencia regulada de Enthoven, oriente cada vez más las reformas de los sistemas de salud, derivadas de los altos costos de los servicios médicos, la crisis fiscal y el ajuste estructural promovido por las agencias financieras multilaterales. Crece el predominio de una posición liberal contractualista, regulada y acompañada del subsidio a la demanda de los más pobres, a pesar de los desarrollos desiguales (35).

La propuesta de construcción democrática

Es posible continuar en la tendencia. El debate regional pasa por una nueva fase de confrontación de opciones, a partir del reconocimiento del problema de equidad en salud como un asunto fundamental de interés global (22). Lo más probable es que no todos los países introduzcan los elementos de la posición predominante. Pero también vale la pena darse la oportunidad de explorar nuevas vías, por lo menos construidas desde la interacción de posiciones en culturas de gran diversidad como las latinoamericanas. 
Para avanzar en tal sentido, se propone una posición pluralista, en el sentido de la construcción democrática, y no del liberalismo político (36). Tal posición pluralista debe partir de la conciencia de la historicidad de la justicia social y reconocer el malestar que genera la persistencia de las injusticias, innegables en nuestros países desde cualquier posición valorativa. Desde allí, propiciar el debate público sobre lo que se entiende por salud y por equidad, en escenarios legítimos y con reglas acordadas. En tal debate, también se requiere reconocer las desigualdades de poder, a la hora de expresar, identificar, proponer y transformar las condiciones que generan las inequidades (37). Y contar con referentes de otros contextos, para aportar a la construcción de una justicia global, como ha pretendido ser el enfoque de los derechos humanos interdependientes. Desde la posición democrática es posible asumir el reto de cuestionar los predominios y las hegemonías para transformar, sin caer en el vértigo del salto al vacío •

\section{REFERENCIAS}

1. Quevedo E, Hernández M. La articulación del conocimiento básico biológico y social en la formación del profesional de la salud: una mirada desde la historia. En: Rodríguez Ml., ed. Lo biológico y lo social. Su articulación en la formación del personal de salud. Serie Desarrollo de Recursos Humanos № 101. Washington, D.C.: Organización Panamericana de la Salud; 1994: 13-34.

2. Peter F, Evans T. Dimensiones éticas de la equidad en salud. En: Evans T, Whitehead M, Diderichsen F, Bhuiya A, Wirth M. Desafío a la falta de equidad en la salud. De la ética a la acción. Washington: OPS/OMS, Fundación Rockefeller; 2002: 27-35

3. Barry B. Political Argument. Berkeley: University of California Press; 1990.

4. Culyer A, Wagstaff A. Equity and equality in health and health care. J Health Econ 1993; 12: 431457.

5. Whitehead M. The concepts and principles of equity in health. Int J Health Serv 1992; 22: 429-445.

6. Braveman P, Gruskin S. Defining equity in health. J Epid Com Health 2003; 57: 254-258.

7. Gracia D. ¿Qué es un sistema justo de programas de salud? Principios para la asignación de recursos escasos. Bol Of Sanit Panam 1990; 108 (5-6): 570-585.

8. McIntyre A. Alter Virtue. Notre Dame: University of Notre Dam Press; 1981.

9. Nozick R. Anarchy, State and Utopia. New York: Basic Books; 1974. p. 149-153.

10. Hernández M. Neoliberalismo en salud: desarrollos, supuestos y alternativas. En: Restrepo DI., ed. La falacia neoliberal. Crítica y alternativas. Bogotá: Vicerrectoría Académica y Sede Bogotá, Universidad Nacional de Colombia; 2003. p. 347-361.

11. Mill JS. Utilitarism on Liberty. Considerations on representative government. London: Everyman; 1994. p. 43-67.

12. Banco Mundial. Invertir en salud. Informe sobre el desarrollo mundial 1993. Washington DC: Banco Mundial; 1993.

13. Murray CJL, López AD, Jamison DT. La carga global de enfermedad en 1990: resumen de los resultados, análisis de la sensibilidad y orientaciones futuras. Bol Of Sanit Panam 1995; 118 (5): 510-527.

14. Murray CJL, Kreuser J, Whang W. El análisis de la efectividad en función del costo y la elección de políticas: la inversión en los sistemas de salud. Bol Of Sanit Panam 1995; 119 (1): 2851.

15. Gadikou EE, Murria CJL, Frenk J. Defining and measuring health inequality. Bull World Health Organ 2000; 78 (1): 42-54. 
16. Organización Mundial de la Salud-OMS. Informe sobre la salud en el mundo 2000. Mejorar el desempeño de los sistemas de salud. Ginebra: OMS; 1993.

17. Rawls J. Teoría de la justicia. Trad. María Dolores González. México DF: Fondo de Cultura Económica; 1997 [1971].

18. Rawls J. Justice as Fairness: Political not Metaphysical. Phil Pub Affaire 1985; 14 (3): 223-251.

19. Rawls J. Las libertades fundamentales y su prioridad. En: McMurrin SM. ed. Libertad, igualdad y derecho. Barcelona: Ariel; 1988: 9-90.

20. Daniels N. Just Health Care. Cambridge MA: Cambridge University Press; 1985.

21. Fried C. Rights and Health Care - Beyond Equity and Efficiency. New England J Med 1975; 293 (5): 241-245.

22. Commission on Social Determinants of Health-CSDH. Achieving Health Equity: from root causes to fair outcomes. Interim statement. Geneva: CSDH, World Health Organization; 2007.

23. Sen A. ¿lgualdad de qué? En: Mc.Murrin SM. ed. Libertad, igualdad y derecho. Barcelona: Ariel; 1998 [1979]: p. 133-156.

24. SenA. Health Equity: Perspectives, Measurability, and Criteria. In: Evans T, Whitehead M, Diderichsen F, Bhuiya A, Wirth M. Challenging Inequities in Health. From Ethics to Action. New York: Oxford University Press; 2001. p. 69-75.

25. Enthoven AC. Consumer Choice Health Plan: ANacional Insurance Proposal Based on Regulated Competition in Private Sector (two parts). N Engl J Med 1978 March 13th and 30th; 298 (12, 13): 650-658; 709-720.

26. Restrepo M. La reforma a la seguridad social en salud de Colombia y la teoría de la competencia regulada. En: Titelman D, Uthoff A. eds. Ensayos sobre el financiamiento de la seguridad social en salud. Los casos de: Estados Unidos, Canadá, Argentina, Chile, Colombia. Vol. 2. Santiago de Chile: CEPAL, Fondo de Cultura Económica; 2000. p. 659-726.

27. Elias N. Hacia una teoría de los procesos sociales. En: Elias N. La civilización de los padres y otros ensayos. Compilación, presentación y traducción de Vera Weiler. Bogotá: Norma, Editorial Universidad Nacional; 1998: 139-197

28. De Swaan A. A cargo del Estado. Barcelona: Pomarés-Corredor; 1992. p. 179-254.

29. Polanyi K. La gran transformación. Critica del liberalismo económico. Madrid: La Piqueta; 1997.

30. Castel R. Las metamorfosis de la cuestión social. Una crónica del salariado. Buenos Aires: Paidós; 1997.

31. Navarro V. Why some Countries have National Health Insurance, others have National Health Services, and the U.S. has neither?. Soc Sci Med 1989; 28 (9): 887-898.

32. Roemer M. Los modelos de servicios de salud en América Latina: Perú, Costa Rica, México, Brasil y Chile. En: Roemer M. Perspectiva Mundial de los Sistemas de Salud. México DF: Siglo XXI; 1980. p. 106-130.

33. Hernández M. El enfoque sociopolítico para el análisis de las reformas sanitarias en América Latina. Revista Facultad Nacional de Salud Pública. 2001, enero-junio; 19 (1): 57-70.

34. Londoño JL, Frenk, J. Structured Pluralism: towards a New Model for Health System Reform in Latin America. Washington DC: Technical Department for Latin America and the Caribbean, the World Bank; 1995.

35. Infante A, De la Mata I, López-Acuña D. Reforma de los sistemas de salud en América Latina y el Caribe. Situación y Tendencias. Rev Pan Salud Pub 2000; 8 (1/2): 13-20.

36. Hernández M. La Bioética y el pluralismo ético. Rev Ger Pol Salud 2001; 1: 74-85.

37. Hernández M, Ochoa D, Novoa E. Del capital social a la construcción de lo público. Revista Trans 2001; $1: 12-41$ 